\title{
Outcomes of venopulmonary arterial extracorporeal life support as temporary right ventricular support after left ventricular assist implantation
}

\author{
Sajad Shehab, BMedSci, PhD, ${ }^{\mathrm{a}, \mathrm{b}}$ Sriram Rao, MBBS, FRACP, ${ }^{\mathrm{b}}$ Peter Macdonald, MD, PhD, FRACP, ${ }^{\mathrm{b}, \mathrm{c}, \mathrm{d}}$ \\ Phillip J. Newton, RN, PhD, FAHA, ${ }^{a}$ Phillip Spratt, MBBS, FRACS, ${ }^{b}$ Paul Jansz, BMed, PhD, FRACS, ${ }^{b}$ and \\ Christopher S. Hayward, BMedSci, MD, FRACP ${ }^{\mathrm{b}, \mathrm{c}, \mathrm{d}}$
}

\section{ABSTRACT}

Objectives: We report our experience with temporary postoperative venopulmonary arterial extracorporeal life support as short-term right ventricular support in patients with biventricular failure undergoing HeartWare (HeartWare Inc, Framingham, Mass) left ventricular assist device implantation and compared these outcomes with isolated left ventricular assist device support and long-term biventricular assist device support.

Methods: A total of 112 consecutive patients were studied, 75 with the isolated HeartWare left ventricular assist device, 23 with a concomitant left ventricular assist device and venopulmonary arterial extracorporeal life support, and 14 with durable biventricular assist device support. The decision concerning shortterm or durable biventricular support was based on the clinical characteristics, echocardiography, and hemodynamic profile.

Results: Patients who required venopulmonary arterial extracorporeal life support after left ventricular assist device insertion required the greatest support preoperatively and were more likely to have Interagency Registry for Mechanical Assisted Circulatory Support level 1 (left ventricular assist device 19\%, venopulmonary arterial extracorporeal life support $48 \%$, biventricular assist device $57 \%$, $P<.001$ ). They were more likely to require preoperative mechanical support (left ventricular assist device $9 \%$, venopulmonary arterial extracorporeal life support $43 \%$, and biventricular assist device $29 \%, P<.001$ ) or preoperative ventilation $(9 \%, 38 \%$, and $21 \%$, respectively, $P<.05)$. Preoperative echocardiographic and hemodynamic parameters were more similar to those requiring isolated left ventricular assist device, with patients requiring durable biventricular assist device support more likely to have elevated right atrial pressure (left ventricular assist device $14.3 \pm 6.7$ vs venopulmonary arterial extracorporeal life support $13.6 \pm 4.8$ vs biventricular assist device $18.7 \pm 6.0 \mathrm{~mm} \mathrm{Hg}, P<.05$ ); right atrial/pulmonary capillary wedge pressure ratio $(0.53 \pm 0.23$ vs $0.51 \pm 0.17$, vs $0.69 \pm 0.22$, respectively, $P<.02)$; and tricuspid regurgitation grade $(1.7 \pm 1.5$ vs $1.7 \pm 1.6$ vs $2.8 \pm 1.6$, respectively, $P<.01$ ). One-year survival was $84 \%$ for those with an isolated left ventricular assist device compared with $62 \%$ for those with venopulmonary arterial extracorporeal life support and $64.3 \%$ for biventricular assist device.

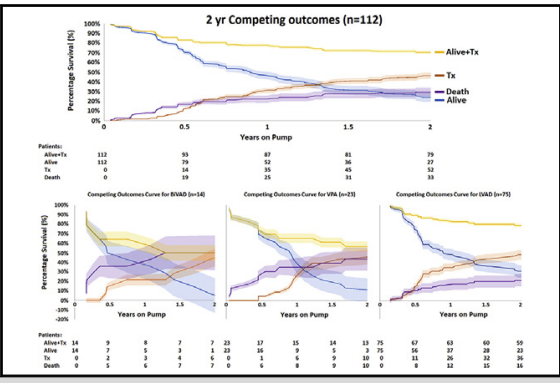

BiVAD, VPA, LVAD, BiVAD censored, VPA censored, and LVAD censored.

\section{Central Message}

In patients who may otherwise require biventricular support, planned concomitant VPAECLS provides acceptable perioperative RV support.

\section{Perspective}

Patients with biventricular failure represent a high-risk group for isolated LVAD implantation. Advances in cannulation techniques have broadened applications and improved the risk-benefit profile of VPA-ECLS, making it a feasible alternative to traditional temporary paracorporeal RVADs. Rekindled interest in ECLS therapy has direct implications for patient care, resource use, and clinical training.

See Editorial Commentary page 2153.

\footnotetext{
From the ${ }^{\mathrm{a}}$ Centre for Cardiovascular and Chronic Care, University of Technology Sydney, Sydney, New South Wales, Australia; ${ }^{\mathrm{b}}$ Cardiology Department, St Vincent's Hospital, Sydney, New South Wales, Australia; ${ }^{\text {c} V i c t o r ~ C h a n g ~ C a r d i a c ~}$ Research Institute, Sydney, New South Wales, Australia; and ${ }^{\mathrm{d}}$ School of Medicine, University of New South Wales, Kensington, New South Wales, Australia.

S.S. is supported by an Australian Postgraduate Award from the Australian Government.

Received for publication May 4, 2017; revisions received April 30, 2018; accepted for publication May 21, 2018; available ahead of print July 17, 2018.
} Address for reprints: Christopher S. Hayward, BMedSci, MD, FRACP, Cardiology
Department, St Vincent's Hospital, 390 Victoria St, Darlinghurst, New South
Wales, 2010 Australia (E-mail: cshayward@stvincents.com.au).
0022-5223/ $\$ 36.00$
Crown Copyright $@ 2018$ Published by Elsevier Inc. on behalf of The American As-
sociation for Thoracic Surgery
https://doi.org/10.1016/j.jtcvs.2018.05.077 


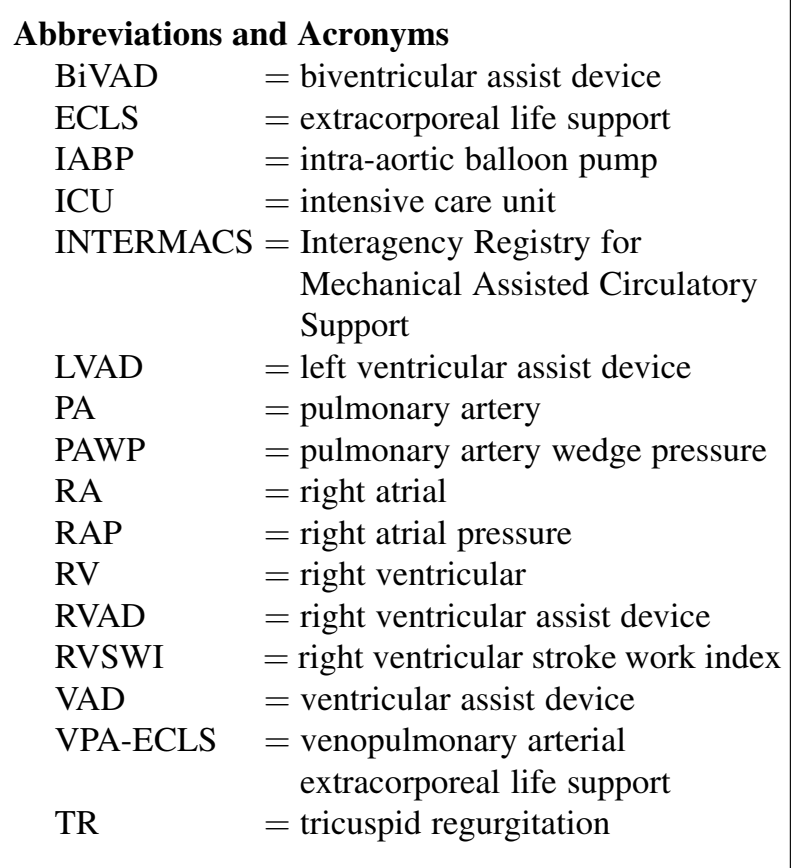

Scanning this QR code will take you to the article title page.

Conclusions: In critically unwell patients requiring temporary biventricular support, planned venopulmonary arterial extracorporeal life support provides acceptable outcomes, similar to durable biventricular assist device support. Requirement for venopulmonary arterial extracorporeal life support is more determined by the level of preoperative acuity than echocardiographic or hemodynamic parameters. (J Thorac Cardiovasc Surg 2018;156:2143-52)

Implantation of continuous-flow left ventricular assist devices (LVADs) has significantly improved survival for patients with end-stage heart failure awaiting heart transplantation. ${ }^{1,2}$ Despite improvements, concomitant right heart failure requiring right ventricular (RV) support $^{3}$ develops in $20 \%$ to $30 \%$ of patients with LVADs. $\mathrm{RV}$ failure after LVAD implantation is associated with poor clinical outcomes and high rates of mortality. Although some patients may improve with pharmacologic therapies such as prostaglandins, nitric oxide, and ionotropic agents, ${ }^{4}$ others require mechanical circulatory support, ${ }^{5}$ which includes intra-aortic balloon pumps (IABPs), right ventricular assist devices (RVADs), and extracorporeal life support (ECLS). Patients at high risk of concomitant RV failure should be supported with early biventricular assist device (BiVAD) support because delayed RVAD after LVAD implantation with current devices has led to poor outcomes. ${ }^{6,7}$

Because of the better outcomes with isolated LVAD, durable BiVAD support is undertaken only in irretrievable cases. Short-term RVAD support has been successfully performed with various VAD models yielding similar results, ${ }^{7,8}$ but repeat surgery for explantation and the limitations associated with paracorporeal systems increase the risk of mortality from temporary RVADs. ${ }^{9}$ The venopulmonary arterial (VPA) ECLS, a modified version of a cardiopulmonary bypass circuit, is a well-established technique and is routinely used in cardiac surgery. ${ }^{10}$ Recent advances in cannulation techniques have resulted in an improved risk-benefit profile and broadened potential applications of VPA-ECLS, ${ }^{11}$ making it a feasible alternative to traditional temporary paracorporeal RVADs. These improvements may allow for restored RV function and thus prevent the need for durable BiVAD support. However, there is little information concerning decision pathways and long-term clinical outcomes in patients receiving temporary VPA-ECLS in combination with LVADs. ${ }^{5}$ The aim of this study is to report our experience with VPA-ECLS as temporary RV support in patients undergoing LVAD implantation and compare the outcome of this approach with that of isolated LVAD only and with an initial surgical strategy of BiVAD support.

\section{MATERIALS AND METHODS \\ Study Population}

We reviewed all consecutive patients undergoing implantation of mechanical circulatory support at a single quaternary Heart Transplant Center between 2007 and 2016. All patients received implants as a bridge-totransplantation strategy and were followed up until heart transplantation or death on mechanical support. The data collection in this study was approved by the local Human Research Ethics Committee. All patients had advanced heart failure classified according to the Interagency Registry for Mechanically Assisted Circulatory Support (INTERMACS Score 1-3).

\section{Criteria Guiding Implant Strategy}

The implant strategy for planned, durable BiVAD support was based on a combination of hemodynamic parameters of impaired RV function (right ventricular stroke work index $[\mathrm{RVSWI}] \leq 0.30 \mathrm{~mm} \mathrm{Hg} \mathrm{L} / \mathrm{m}^{2},{ }^{12}$ right atrial pressure $[\mathrm{RAP}] \geq 15 \mathrm{~mm} \mathrm{Hg},{ }^{4}$ right atrial $[\mathrm{RA}] /$ pulmonary artery wedge pressure (PAWP) ratio $\geq 0.63$, transpulmonary gradient $>12 \mathrm{~mm} \mathrm{Hg}$ ), and echocardiographic markers (RV dilatation score $\geq 3$, RV systolic impairment score $\geq 3$, and tricuspid regurgitation [TR] grade $\geq 3$ ). Echocardiographic markers were calculated using previously described methodology, ${ }^{13}$ assigning numeric codes according to valvular and ventricular abnormalities ranging from 0 (normal or trivial) to 5 (severe regurgitation or impairment). In patients with mild-moderate RV impairment or better (RV impairment score $<3$ ) and high probability of weaning of temporary RVAD postimplantation (TR grade $<3$, RV dilatation score $<3$, RAP $<15$, RVSWI $\leq 0.30$, RA/PAWP $<0.63$ ), concomitant LVAD with shortterm VPA-ECLS was used. 


\section{Implantation Procedure}

As previously described, ${ }^{14}$ VPA-ECLS with the MAQUET Rotaflow centrifugal pump (Rastatt, Germany) was instituted using femoral venous cannulation withdrawing blood to the pump. Outflow from the MAQUET Rotaflow was then attached to an 8-mm Dacron graft tunneled through the precordium and sutured end to side to the proximal pulmonary artery (PA) trunk, enabling separation from ECLS to be conducted in the intensive care unit (ICU) without returning to the operating room. The ECLS circuit has an integrated oxygenator (MAQUET PLS-I Oxygenator) and was used for all cases. The left HeartWare (HeartWare Inc, Framingham, Mass) VAD was implanted via sternotomy into the apex of the left ventricle, with the outflow graft attached to the ascending aorta in all 112 patients. The right HeartWare VAD implantation procedure for our BiVAD cohort was in an RA and or RV configuration and has been described. ${ }^{15}$

\section{Anticoagulation}

Postoperative anticoagulation with intravenous heparin began 8 to 12 hours after surgery, and only after blood loss from drain tubes reached less than $50 \mathrm{~mL} / \mathrm{h}$, to derive an activated partial thromboplastin time between 50 and 75 seconds. Oral anticoagulation in combination with aspirin antiplatelet therapy began after the removal of mediastinal or chest drain tubes. To detect clinically significant hemolysis, free hemoglobin, bilirubin, and lactate dehydrogenase blood levels were analyzed daily during ICU stay and then on a monthly basis for outpatient visits.

\section{Postoperative Management}

All patients received a standardized protocol for postoperative care for VAD support. This included prophylactic broad-spectrum antibiotics for the first 3 days postimplantation and close monitoring and correction of coagulation profiles. Within 1 week of restoring a patient's hemodynamic profile, echocardiography was performed to determine structural improvements and pump settings.

\section{Venopulmonary Arterial Extracorporeal Life Support Weaning and Explantation Process}

VPA-ECLS removal was considered once patients showed improvements in their clinical status, with stabilization of inotrope dose and gradual weaning of pressors with improving renal function. Echocardiographic and hemodynamic markers alone were not relied on to determine weaning. The removal was performed after gradual reduction of the VPA-ECLS flow to $2 \mathrm{~L} / \mathrm{min}$ and maintained for 2 hours to assess clinical stability. Inotropic agents were maintained before the weaning process. Excess portions of the $8-\mathrm{mm}$ Dacron graft were removed before it was heavily ligated, clamped, and oversewn. The graft was retracted back into the chest, and the skin incision was closed.

\section{Outcomes}

Postoperative outcomes measured in this study include length of stay, ICU length of stay, survival at 30 days and 1 year, and duration of support. Postoperative clinical outcomes such as estimated glomerular filtration rate and adverse events such as infection and bleeding were also measured. All postoperative outcomes were censored at the time of transplantation.

\section{Statistical Analysis}

Statistical analysis was performed using SPSS version 11.5 for Windows (SPSS Inc, Chicago, Ill). Results are presented as mean \pm standard deviation for continuous data and percentages for categoric data. Differences between demographic data for the 3 groups were analyzed using analysis of variance, with post hoc Bonferroni correction. Nonparametric Mann-Whitney $U$ test was used to analyze both continuous and categoric data. Paired-samples $t$ test was used to assess associations between preoperative and postoperative outcomes and adverse events. Time to death and time to transplantation were analyzed as competing outcomes. Adjusted $P$ values for survival were obtained by Cox regression analysis.

\section{RESULTS}

\section{Patient Characteristics}

Between April 2007 and August 2016, 112 consecutive patients (90 male) underwent implantation of the HeartWare VAD for isolated left ventricular support $(\mathrm{n}=75)$, concomitant LVAD and short-term VPA-ECLS $(\mathrm{n}=23)$, and initial BiVAD support $(\mathrm{n}=14)$. Patient age ranged from 12 to 76 years (mean $51 \pm 14$ years), and body mass index ranged from 16 to $43 \mathrm{~kg} / \mathrm{m}^{2}$ (mean $26 \pm 6 \mathrm{~kg} / \mathrm{m}^{2}$ ). Before implantation, all patients were on intravenous inotropes therapy; 35 were on IABP and 21 were on venoarterial ECLS. Four patients ( 2 in the VPA-ECLS and 2 in the BiVAD group) received delayed RV support 3 to 10 days post-LVAD support.

As shown in Table 1 and Figure 1, there were significant differences observed in INTERMACS class, preimplant mechanical, and ventilatory support among the 3 groups. Patients with BiVADs had significantly higher RAP than the VPA-ECLS $(P<.05)$ and LVAD $(P<.05)$ cohorts. This is also observed in the RA/PAWP ratio of the BiVAD and LVAD $(P<.02)$ group compared with VPA-ECLS. The BiVAD cohort had greater RV impairment compared with the LVAD cohort $(P<.05)$, whereas RV dilatation was significantly different among all groups $(P<.05)$. Generally, both the echocardiographic and hemodynamic indices of the VPA-ECLS group were more similar to the isolated LVAD cohort, than the BiVAD cohort (Figure 1). Compared with isolated LVAD, patients in the VPA-ECLS and BiVAD cohort had marked hepatic congestion demonstrated by their bilirubin levels $39.4 \pm 29.6 \mu \mathrm{mol} / \mathrm{L}$ and $35.1 \pm 25.3 \mu \mathrm{mol} / \mathrm{L}$, respectively $(P<.01)$. Adjusted Cox regression analysis showed age at implant $(P=.034)$, gender $(P=.005)$, liver dysfunction (albumin $[P=.029]$ and bilirubin $[P=.036]$ ) and poor preoperative RV dilatation $(P=.025)$, and $\mathrm{RV}$ impairment $(P=.021)$ to be significantly associated with increased risk of mortality. No other clinical characteristics were significantly different among the 3 groups.

Isolated LVAD was generally planned in the absence of clinical evidence of right heart failure. As discussed earlier, moderate RV dilatation or RV impairment (score $>2$ ) was associated with an increased risk of mortality, but there was no difference in preoperative RA $(15.0 \pm 6.6$ vs $11.1 \pm 8.5 \mathrm{~mm} \mathrm{Hg}, \mathrm{RV}$ score $>2$ vs $\leq 2, P=.14$ ) or PCWP ( $27.6 \pm 6.3$ vs $25.3 \pm 5.2 \mathrm{~mm} \mathrm{Hg}, P=.33)$ between the 2 groups. When preoperative hemodynamic indices were examined, there was no difference in survival according to RAP, RA/PCWP ratio, transpulmonary gradient, or RVSWI. In general, there was only limited agreement between echocardiographic and hemodynamic indices, and the final decision among the isolated LVAD and short and long-term BiVADs was made after multidisciplinary team 
TABLE 1. Preoperative characteristics

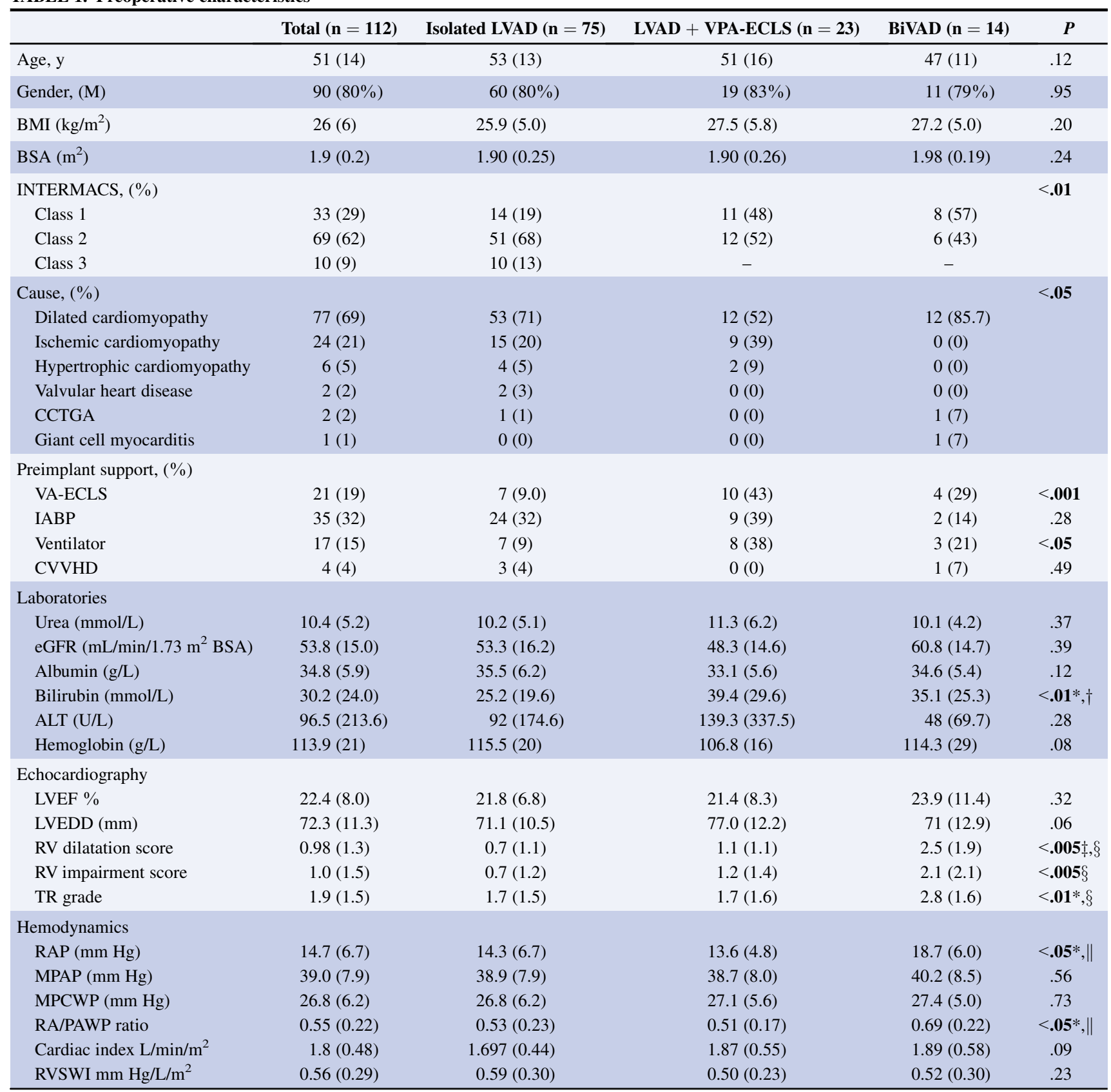

Continuous variables shown as mean (standard deviation). Statistical significance is indicated in bold. $L V A D$, Left ventricular assist device; VPA-ECLS, venopulmonary arterial extracorporeal life support; BiVAD, biventricular assist device; BMI, body mass index; BSA, body surface area; INTERMACS, Interagency Registry for Mechanical Assisted Circulatory Support; $C C T G A$, congenital corrected transposition of the greater arteries; VA-ECLS, venoarterial extracorporeal life support; IABP, intra-aortic balloon pump; $C V V H D$, continuous veno-venous hemodialysis; $A L T$, alanine amino transferase; $L V E F$, left ventricular ejection fraction; $L V E D D$, left ventricular end-diastolic diameter; $R V$, right ventricle; $T R$, tricuspid regurgitation; $R A P$, right atrial pressure; $M P A P$, mean pulmonary artery pressure; $M P C W P$, mean pulmonary wedge pressure; $R A / P A W P$, right atrial/pul-

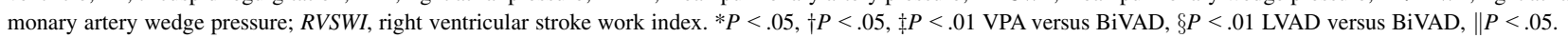

review, taking into account clinical status, as well as renal and hepatic function.

\section{Postoperative Outcomes}

We report outcomes from a cumulative VPA-ECLS support duration across 23 patients of 6962 days with a mean duration of support of $302.7 \pm 211.0$ days. Total $(\mathrm{n}=112)$ 1-year survival was $78 \%$, with the highest survival achieved with isolated LVAD (84\%) followed by VPA-ECLS $(65 \%)$ and BiVAD (64\%). Patients requiring RV support had similar mortality regardless of configuration in raw and Cox-adjusted survival curves (Figure 2). 

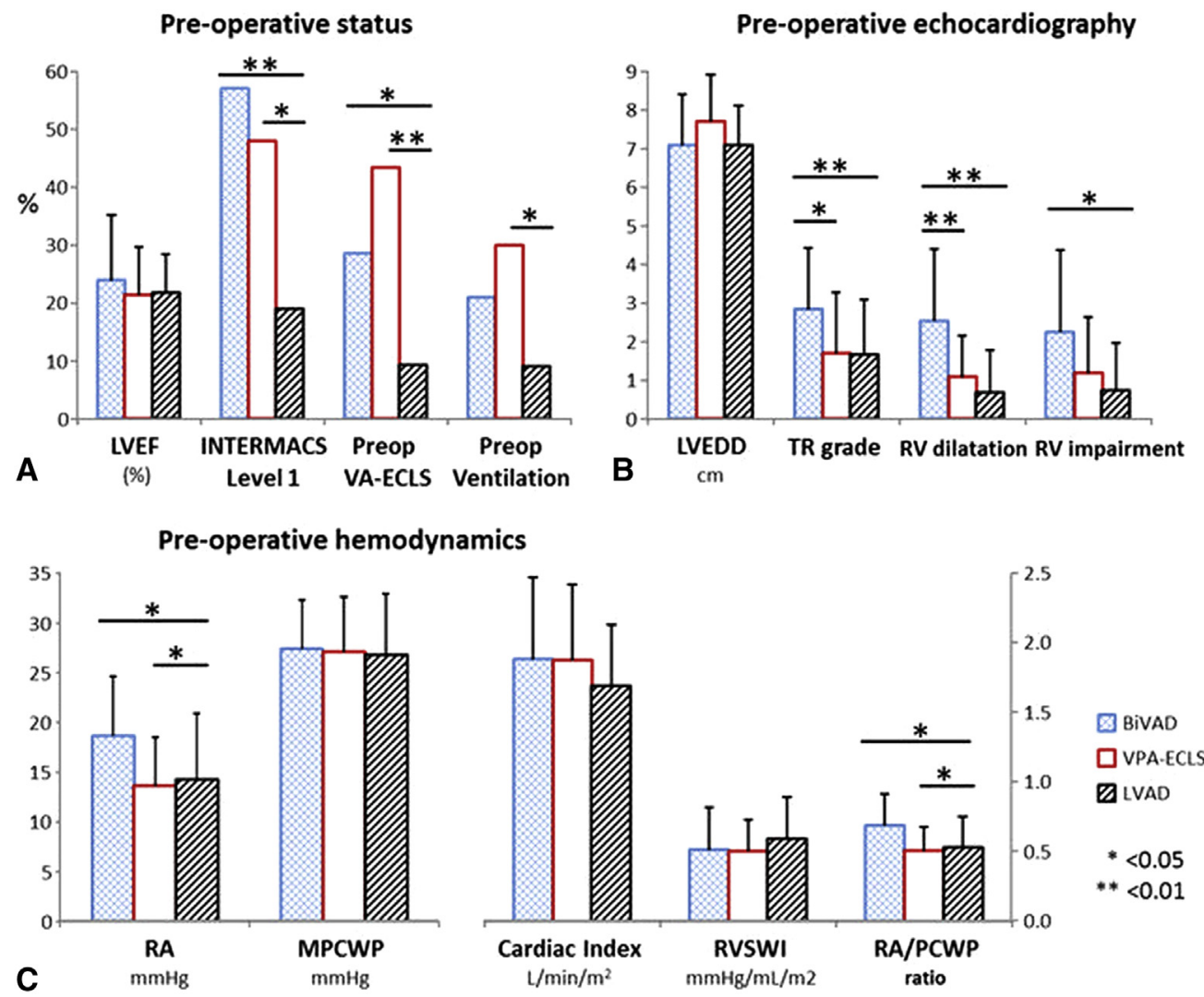

FIGURE 1. Preoperative characteristics according to implant strategy. A, Preoperative status, (B) echocardiographic, and (C) hemodynamics parameters for all support configurations. $L V E F$, Left ventricular ejection fraction; INTERMACS, Interagency Registry for Mechanical Assisted Circulatory Support; Preop, preoperative; VA-ECLS, venoarterial arterial extracorporeal life support; $L V E D D$, left ventricular end-diastolic dimension; TR, tricuspid regurgitation; $R V$, right ventricle; $R A$, right atrial; $M P C W P$, mean pulmonary capillary wedge pressure; $R V S W I$, right ventricular stroke work index; $P C W P$, pulmonary capillary wedge pressure; BiVAD, biventricular assist device; $L V A D$, left ventricular assist device.

Perioperative (30-day) survival was significantly reduced in the VPA-ECLS $(87 \%)$ group when compared with the LVAD group $(100 \%)$ and BiVAD group $(100 \%)$ $(P<.005)$. A total of 34 patients (LVAD $=17$, $\operatorname{BiVAD}=7$, and VPA-ECLS $=10$ ) died during VAD support $(34 / 112[30 \%])$, typically early in the support period. Fifteen patients (15/34 [44\%]) died of neurologic complications, 11 patients $(11 / 34$ [32\%]) died of multiple organ failure, 6 patients $(6 / 34[18 \%])$ died of sepsis (unrelated to driveline), 1 patient (1/34 [3\%]) died of cancer, and $1 \mathrm{pa}-$ tient $(1 / 34[3 \%])$ died of accidental damage to driveline. A total of 63 patients $(63 / 112[56 \%])$ were successfully bridged to transplantation after a mean of $418 \pm 292$ days of support, and 15 patients remain supported after a mean $586 \pm 515$ days after implantation. Competing outcomes analysis curves for each of the cohorts is shown in Figure 3.

At 30 days, serum creatinine improved from $123 \pm 37 \mu \mathrm{mol} / \mathrm{L}$ to $94 \pm 85 \mu \mathrm{mol} / \mathrm{L}$, and estimated glomerular filtration rate improved from $46 \pm 11 \mathrm{~mL} / \mathrm{min}$ (body surface area corrected) to $71 \pm 24 \mathrm{~mL} / \mathrm{min}$ (body surface area corrected). TR grade remained significantly greater in the BiVAD group compared with the LVAD $(P<.05)$ groups at 30 days. Longer ICU length of stay was observed in patients on BiVAD support and VPA-ECLS compared with those supported with isolated LVAD $(P<.01)$. Hospital discharge after LVAD, BiVAD, and VPA-ECLS implant was successfully achieved in $91 \%, 71 \%$, and $86 \%$ of patients, respectively.

\section{Adverse Events}

Adverse events during VAD support are shown in Table 2 and were classified according to the INTERMACS definitions. LVAD thrombus occurred in both the LVAD and VPA-ECLS cohorts (5\% and 9\%, respectively). No cases of LVAD thrombus occurred in the BiVAD cohort. RVAD thrombus ( 3 on RV support and 1 on RA support) developed in 4 patients $(29 \%)$. A total of 49 patients $(44 \%)$ had infectious complications including driveline infections. Sternal wound sepsis developed in 9 patients $(8 \%)$, driveline infection (without positive blood cultures) developed in 14 patients $(13 \%)$, and positive blood cultures (in the absence of a driveline infection) were noted in 37 patients (33\%). Of these patients, 20 had multiple infectious complications. Infection rates were significantly higher in the BiVAD and 

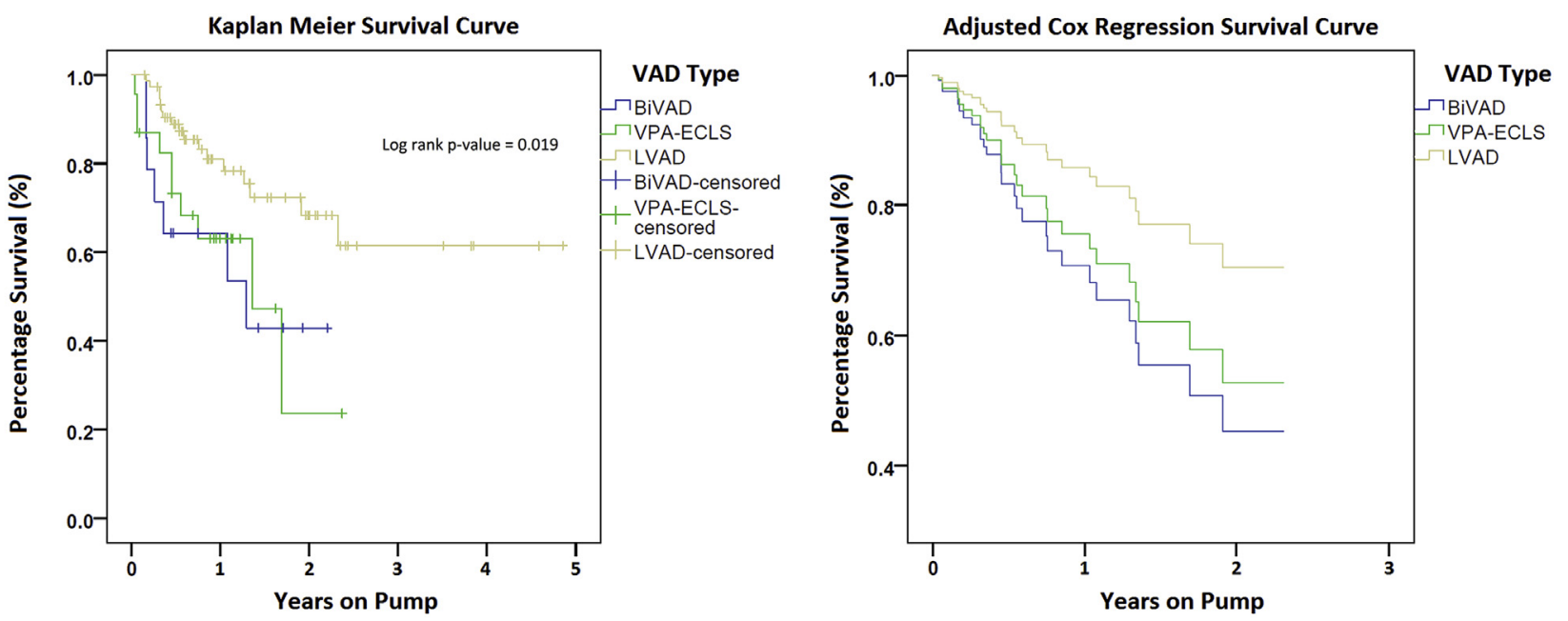

\begin{tabular}{|c|c|c|c|c|c|c|}
\hline BiVAD & 14 & 8 & 7 & - & - & - \\
\hline $95 \% \mathrm{Cl}$ upper limit & & 0.771 & 0.579 & & & \\
\hline $95 \%$ Cl lower limit & & 0.515 & 0.279 & & & \\
\hline VPA-ECLS & 23 & 15 & 13 & - & - & - \\
\hline $95 \% \mathrm{Cl}$ upper limit & & 0.736 & 0.422 & & & \\
\hline $95 \%$ Cl lower limit & & 0.526 & 0.052 & & & \\
\hline LVAD & 75 & 63 & 59 & 58 & 58 & 58 \\
\hline $95 \% \mathrm{Cl}$ upper limit & & 0.861 & 0.756 & 0.708 & 0.708 & 0.708 \\
\hline $95 \%$ Cl lower limit & & 0.759 & 0.610 & 0.522 & 0.522 & 0.522 \\
\hline
\end{tabular}

FIGURE 2. Raw Kaplan-Meier and adjusted competing-risk Cox regression survival curve for isolated LVAD, initial BiVAD, and concomitant VPA-ECLS and LVAD therapy. Survival curve adjusted for preoperative variables and support configuration. BiVAD $\mathrm{n}=14$, VPA-ECLS $\mathrm{n}=23$, and LVAD $\mathrm{n}=75$. $V A D$, Ventricular assist device; $B i V A D$, biventricular assist device; $L V A D$, left ventricular assist device; $V P A-E C L S$, venopulmonary arterial extracorporeal life support; $C I$, confidence interval.

VPA-ECLS groups compared with isolated $\operatorname{LVAD}(P<.01)$. Acute renal failure requiring hemodialysis occurred in 19 patients $(17 \%)$, of whom 15 (5 in LVAD group, 7 in VPA group, and 3 in BiVAD group) successfully recovered. Immediate postoperative acute renal failure developed in 4 patients in the VPA-ECLS cohort and were all successfully resolved within 1 week. Higher rates of neurologic complications observed in the LVAD group (19\% [cerebrovascular accident $13 \%$, transient ischemic attack 5\%]) compared with the VPA-ECLS group (13\%, all cerebrovascular accident) and BiVAD group (14\% [cerebrovascular accident $7 \%$, transient ischemic attack 7\%]) but were not statistically significant. Conversely, elevated rates of respiratory dysfunction were noted in the BiVAD and VPA-ECLS groups compared with the LVAD group, but were not statistically significant $(P=.20)$. Bleeding rates were more common in the isolated LVAD and VPA-ECLS cohorts compared with the BiVAD cohort, but this was not a significant difference. All adverse events reported were events during index hospitalization and from subsequent readmissions.

\section{DISCUSSION}

The study summarizes mechanical circulatory support outcomes with the HeartWare VAD using 3 different configurations, including isolated LVAD support, planned concomitant LVAD, and temporary VPA-ECLS support and long-term BiVAD support (dual HeartWare VADs). Our results with isolated LVADs offer 1-year survival $(84 \%)$ and transplantation rates $(61 \%)$ similar to those reported by other groups using the same device ${ }^{16,17}$ and to that of published registry data. ${ }^{18}$ Our VPA-ECLS cohort is reflective of patients at risk of developing acute RV failure post-LVAD implantation. Nonetheless, the majority of patients were weaned successfully from VPA-ECLS and supported thereafter with isolated LVADs. Although preoperative demographics were similar between all groups, the main determinant for patients requiring shortterm RVAD support was clinical acuity, with a high rate of preoperative mechanical and ventilator support. The hemodynamic features of this cohort, such as RAP and RA/ PAWP, were more similar to those of the isolated LVAD group, whereas other markers such as bilirubin and RVSWI were closely matched with the BiVAD cohort. Echocardiographic markers used as part of the BiVAD selection criteria, such as RV dilatation (score $\geq 3$ ), RV impairment $(\geq 3)$, and TR grade $(\geq 3)$, were lower than what was required for long-term initial BiVAD support but higher than isolated LVAD. The BiVAD cohort was significantly more likely to have structural RV changes of impaired, dilatation, and TR.

When examining the patients who received initial isolated LVAD support, but subsequently died, there was evidence of some degree of right heart impairment evident 

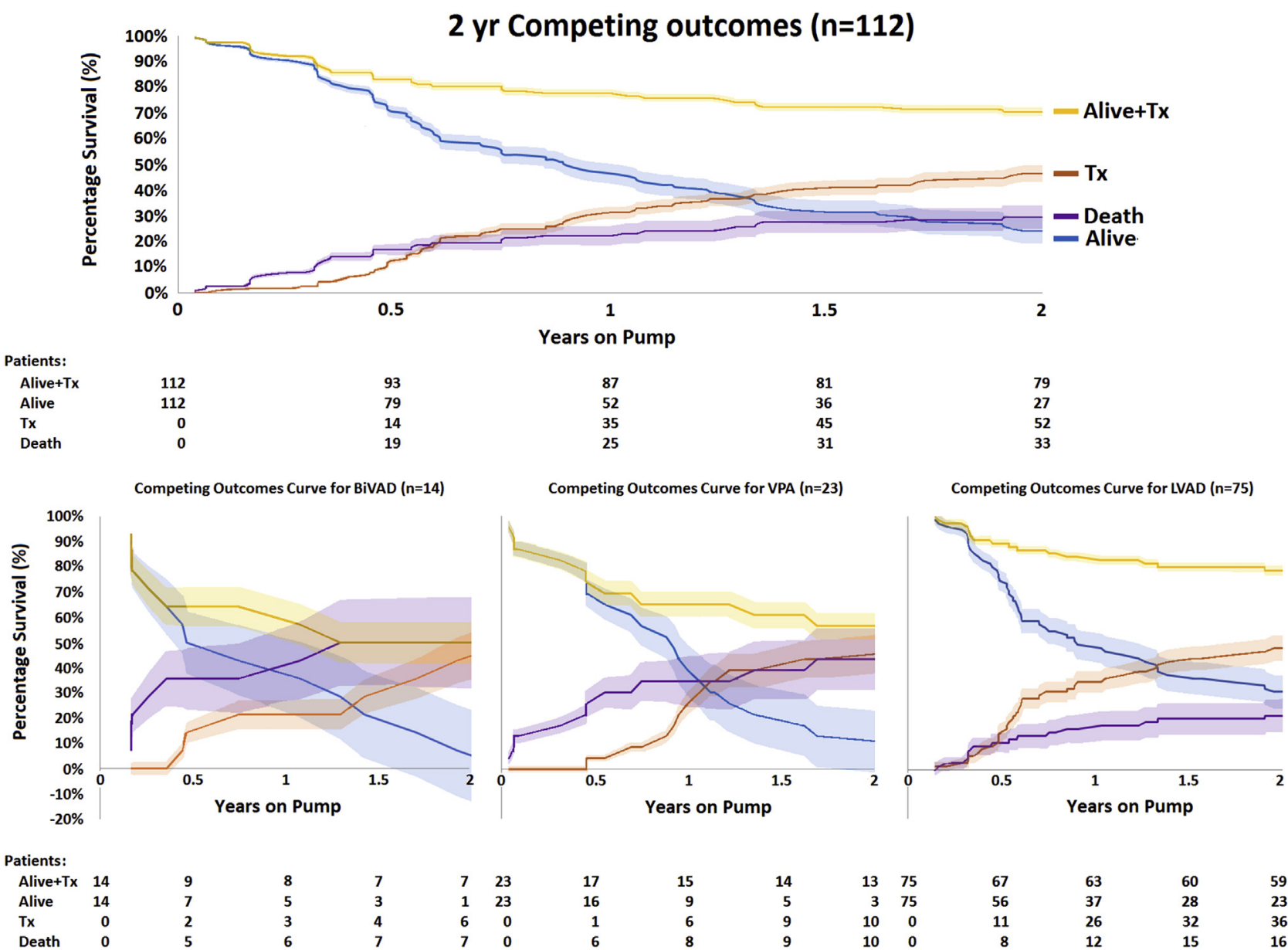

$\begin{array}{rrrrrrr}7 & 23 & 17 & 15 & 14 & 13 & 75 \\ 1 & 23 & 16 & 9 & 5 & 3 & 75 \\ 6 & 0 & 1 & 6 & 9 & 10 & 0 \\ 7 & 0 & 6 & 8 & 9 & 10 & 0\end{array}$

$\begin{array}{rr}67 & 63 \\ 56 & 37 \\ 11 & 26 \\ 8 & 12\end{array}$

$\begin{array}{ll}60 & 59 \\ 28 & 23 \\ 32 & 36 \\ 15 & 16\end{array}$

FIGURE 3. Two-year competing outcomes curve for isolated LVAD, initial BiVAD, and concomitant VPA ECLS and LVAD therapy. Tx, Transplantation; $B i V A D$, biventricular assist device; $V P A$, venopulmonary arterial; $L V A D$, left ventricular assist device.

on post hoc analysis, with a significantly higher rate of RV dilatation or impairment preoperatively. The analysis may be complicated by time bias, in that VPA-ECLS was not used in our hospital until 2009, and patients from before then may have benefited from short-term support. Likewise, BiVAD implants were used only from 2011 at our hospital.

Various configurations of VPA-ECLS, such as RA to PA bypass, have been reported but require reoperation to remove the cannula, increasing the risk of infection and surgical-related bleeding. The same limitations apply to existing paracorporeal RVAD and BiVAD systems. ${ }^{19-21}$ Our institutional configuration of femoral venous cannulation for inflow and PA trunk for outflow enables removal of VPA-ECLS without reoperation, eliminating these risks. A similar procedure was reported by Saeed and colleagues $^{22}$ in 21 patients using the Levitronix CentriMag (Thoratec, Pleasanton, Calif) as temporary RV support. The inflow cannula was inserted percutaneously into the right atrium through the right femoral vein, and the outflow graft was connected to the main PA. In that cohort, 11 patients were successfully weaned off RV support, 2 patients were bridged to transplantation, and 8 patients died. The 1 -year survival was similar between that cohort and the one described $\left(52 \%{ }^{22}\right.$ vs $\left.65 \%\right)$. The lower survival may have been due to oxygenator use at the time of RVAD support. Oxygenators can be helpful in the event of hypoxia or severe lung edema, or in patients on ECLS support before VAD support. ${ }^{23}$ In our study, all patients were standardly supported with an oxygenator (MAQUET PLS-I Oxygenator), whereas only 12 patients $(57 \%)$ were provided in the study by Saeed and colleagues. ${ }^{22}$

A feature of the current cohort is the high proportion of patients with INTERMACS 1, with a high rate of mechanical support before LVAD use and multiorgan dysfunction, known to be risk factors for early mortality. ${ }^{9}$ Furthermore, the chronicity and severity of advanced heart failure result in a state of physiologic frailty. Although frailty was not formally measured in this cohort, it has been shown to 
TABLE 2. Postoperative outcomes, clinical outcomes, and adverse events

\begin{tabular}{|c|c|c|c|c|c|}
\hline & Total $(n=112)$ & Isolated LVAD $(n=75)$ & LVAD + VPA-ECLS $(n=23)$ & $\operatorname{BiVAD}(n=14)$ & $P$ value \\
\hline \multicolumn{6}{|l|}{ Postoperative outcomes } \\
\hline LoS, d (mean) & $37.6(21.7)$ & $33.9(20.2)$ & $46.3(25.6)$ & $41.5(21.2)$ & $<.05^{*}$ \\
\hline ICU LoS, d (mean) & $12.7(12.3)$ & $9.0(7.9)$ & $20.6(12.1)$ & $19.8(20.8)$ & $<.01 \dagger, \ddagger$ \\
\hline Duration of support, $d$ (mean) & $393.0(336.4)$ & $421.4(364.7)$ & $302.7(211.0)$ & $312.6(261.5)$ & .17 \\
\hline TR grade (mean) & $0.8(1.2)$ & $0.8(1.3)$ & $0.3(0.7)$ & $1.6(1.3)$ & $<. \mathbf{0 5} \oint$ \\
\hline 30-d survival $(\%)$ & $109(97)$ & $75(100)$ & $20(87)$ & $14(100)$ & $<.005 \ddagger$ \\
\hline Survived to discharge $(\%)$ & $96(86)$ & $68(91)$ & $19(83)$ & $10(71)$ & .12 \\
\hline $1-y$ survival $(\%)$ & $87(78)$ & $63(84)$ & $15(65)$ & $9(64)$ & .07 \\
\hline Transplanted (\%) & $63(56)$ & $46(61)$ & $11(48)$ & $7(50)$ & .44 \\
\hline Died on support $(\%)$ & $34(30)$ & $17(23)$ & $10(43)$ & $7(50)$ & $<.05$ \\
\hline \multicolumn{6}{|l|}{ Clinical outcomes (30 d) } \\
\hline Urea $(\mu \mathrm{mol} / \mathrm{L})$, mean $(\mathrm{SD})$ & $7.3(4.2)$ & $6.9(3.5)$ & $8.0(4.2)$ & $7.9(4.1)$ & .61 \\
\hline Creatinine $(\mu \mathrm{mol} / \mathrm{L})$, mean $(\mathrm{SD})$ & $88.7(47.7)$ & $85.8(33.0)$ & $89.5(37.1)$ & $97.1(82.8)$ & .87 \\
\hline eGFR $(\mu \mathrm{mol} / \mathrm{L})$, mean $(\mathrm{SD})$ & $66.2(18.8)$ & $65.5(15.9)$ & $62.1(20.6)$ & $69.2(23.4)$ & .49 \\
\hline Albumin $(\mu \mathrm{mol} / \mathrm{L})$, mean $(\mathrm{SD})$ & $35.9(4.7)$ & $36.9(4.6)$ & $34.4(5.5)$ & $33.6(3.5)$ & .08 \\
\hline Bilirubin $(\mu \mathrm{mol} / \mathrm{L})$, mean $(\mathrm{SD})$ & $22.9(49.9)$ & $15.4(11.0)$ & $21.8(11.0)$ & $47.1(15.9)$ & .08 \\
\hline $\operatorname{ALT}(\mathrm{nmol} / \mathrm{L})$, mean $(\mathrm{SD})$ & $25.7(21.5)$ & $25.6(23.0)$ & $23.1(14.9)$ & $27.6(20.7)$ & .92 \\
\hline GGT (mmol/L), mean (SD) & $156.7(119.6)$ & $154.0(131.6)$ & $172.2(122.2)$ & $155.5(73.1)$ & .68 \\
\hline \multicolumn{6}{|l|}{ Adverse events, $(\%)$} \\
\hline LVAD thrombosis & $6(5)$ & $4(5)$ & $2(9)$ & $0(0)$ & .52 \\
\hline RVAD thrombosis & - & - & - & $4(29)$ & NA \\
\hline Bleeding & $26(23)$ & $14(19)$ & $9(39)$ & $3(21)$ & .13 \\
\hline Infection & $49(44)$ & $25(33)$ & $15(65)$ & $9(64)$ & $<.01$ \\
\hline Driveline & $14(13)$ & $9(12)$ & $4(17)$ & $1(7)$ & .64 \\
\hline Sternal wound & $9(8)$ & $4(5)$ & $3(13)$ & $2(14)$ & .32 \\
\hline Blood culture $>0$ & $37(33)$ & $20(27)$ & $11(48)$ & $6(43)$ & .12 \\
\hline Renal dysfunction & $19(17)$ & $8(27)$ & $7(30)$ & $4(29)$ & $<.05$ \\
\hline Neurologic & $19(17)$ & $14(19)$ & $3(13)$ & $2(14)$ & .79 \\
\hline Cerebrovascular accident & $14(13)$ & $10(13)$ & $3(13)$ & $1(7)$ & .81 \\
\hline Transient ischemic attack & $5(4)$ & $4(5)$ & $0(0)$ & $1(7)$ & .49 \\
\hline Respiratory dysfunction & $15(13)$ & $7(1)$ & $5(22)$ & $3(21)$ & .20 \\
\hline
\end{tabular}

Statistical significance is indicated in bold. $L V A D$, Left ventricular assist device; VPA-ECLS, venopulmonary arterial extracorporeal life support; BiVAD, biventricular assist device; $L O S$, length of stay; $I C U$, intensive care unit; $T R$, tricuspid regurgitation; $S D$, standard deviation; $A L T$, alanine amino transferase; $G G T$, gamma-glutamyltransferase; $R V A D$, right ventricular assist device; $N A$, not applicable. ${ }^{*} P<.05, \dagger P<.01$ LVAD versus BiVAD, $\uparrow P<.01$ LVAD versus VPA, $\S P<.05, \| P<.01$ VPA versus BiVAD.

have marked survival implications and is only now prospectively evaluated in all LVAD candidates at our institution.

The VPA-ECLS cohort experienced a higher mortality rate compared with the isolated LVAD cohort $(P<.05)$. This may be due to the higher INTERMACs class and need for preoperative mechanical support with ECLS or IABP, both of which are associated with higher mortality and morbidity before VAD support. ${ }^{24,25}$ As shown in Figure 3, the highest rate of mortality occurred within the early postoperative period. Of the entire VPA-ECLS cohort, 10 patients died during support, of which only 1 death occurred while on concomitant VPA-ECLS support at 23 days because of failure to wean from device. The remaining 9 deaths occurred after VPA-ECLS explantation and restabilization to an isolated LVAD at a mean of $203 \pm 194$ days after VPA-ECLS removal. All other patients were successfully weaned from VPA-ECLS after a mean of $7 \pm 5$ days of support and supported for a further $359 \pm 158$ days. Patients weaned from VPA-ECLS who subsequently died tended to have higher PVR at routine right heart catheterization 3 months after LVAD implant $(131 \pm 75$ vs $295 \pm 108$ dynes.s.cm-5, $P<.001)$. The VPA-ECLS survival is higher than in currently existing venoarterial ECLS published reports ${ }^{11,26}$ and existing registry databases. ${ }^{27}$

Despite the controversy surrounding the efficacy of ECLS, recent improvements in design, technique, and patient selection have resulted in its increased frequency of use. Existing evidence indicates increasing survival with ECLS over the last few decades from 38\% (1990-2000) to $46 \%(2011){ }^{27}$ This is also evident in our VPA-ECLS cohort, with all patients supported after the year 2014 either receiving transplant or remaining alive to date (mean duration of support of $336 \pm 75$ days).

As shown in the adjusted Cox regression analysis, VAD configuration alone does not affect survival. Therefore, the differences seen are explained by the patients' preoperative demographics. Patient selection and early recognition 
of predictive markers of RV failure clearly play an integral role in improving clinical outcomes. Previous studies have revealed a number of preoperative markers of RV failure post-VAD implantation, including INTERMACS score, pre-VAD mechanical support such as ECLS or IABP, albumin or bilirubin values, age, body surface area, and hemodynamics such as RA, mean PA pressure, RA/PAWP, and RVSWI parameters, ${ }^{4,12,28}$ many of which have been supported in the current study.

Our postoperative outcomes have shown a similar duration of support, transplantation, and discharge rates of patients with VPA-ECLS as those with initial BiVAD and isolated LVAD. ICU and hospital length of stays between BiVAD and VPA-ECLS cohorts were comparable, but were significantly higher in the VPA-ECLS cohort compared with isolated LVAD $(P<.01)$ because of higher clinical acuity. The novelty of our BiVAD and VPAECLS strategies is associated with a learning curve, as evident in the VPA-ECLS cohort, in which patients receiving implants after 2013 have a significantly lower ICU length of stay than the total cohort (15.4 \pm 9.6 days vs $20.6 \pm 12.1$ days).

Elevated infection rates were observed in both the BiVAD and VPA-ECLS cohorts compared with isolated LVAD $(P<.01)$, but no significant difference was observed between the types of infection. There were no infectionrelated deaths in the isolated LVAD cohort, but there were 4 in the BiVAD cohort and 1 in the VPA-ECLS cohort. The elevated infection rates observed may be due to the use of 2 pumps in the BiVAD group allowing for 2 loci for infection and due to the separate incision sites (entry and exit site) in the VPA-ECLS cohort. Mediastinal infections can occur after the removal of VPA-ECLS, where the detached graft is retracted into the mediastinum. There was 1 incidence of mediastinitis in our VPA-ECLS cohort that was successfully treated, and the patient successfully underwent transplantation.

Aside from increased risk of infection, retracting the 8$\mathrm{mm}$ graft into the patient chest also increases the risk of pulmonary embolism, and monitoring patients via computed tomography has been recommended. Although we did not follow up with computed tomography scans, none of our patients presented with episodes of pulmonary embolism or bleeding complications associated with retained outflow grafts. The feasibility of leaving the outflow tract has been reported. ${ }^{29}$

\section{Study Limitations}

This study was conducted in a single center and therefore was limited in the number of enrolled patients. The small BiVAD and VPA-ECLS sample size is likely underpowered to detect significant differences between groups and thus limits the study's ability to delineate characteristics that may aid in determining the type of intervention suitable for patients. The consecutive nature of the study cohorts may have resulted in a time bias due to the learning and increased experience with patient selection.

\section{CONCLUSIONS}

In critically unwell patients requiring biventricular support, planned postoperative VPA-ECLS provides acceptable outcomes, similar to durable BiVAD support. The requirement for combined LVAD with VPA-ECLS support is more determined by the level of preoperative acuity than by echocardiographic or hemodynamic parameters.

\section{Conflict of Interest Statement}

C.S.H. has received research funds from HeartWare Inc, unrelated to the current study. C.S.H., P.C.J., and P.S. are consultants for HeartWare Inc. All other authors have nothing to disclose with regard to commercial support.

\section{References}

1. Frazier O, Rose EA, McCarthy P, Burton NA, Tector A, Levin H, et al. Improved mortality and rehabilitation of transplant candidates treated with a long-term implantable left ventricular assist system. Ann Surg. 1995;222:327.

2. Oz M, Argenziano M, Catanese KA, Gardocki MT, Goldstein DJ, Ashton RC, et al. Bridge experience with long-term implantable left ventricular assist devices. Circulation. 1997;95:1844-52.

3. Kaul T, Fields B. Postoperative acute refractory right ventricular failure: inci dence, pathogenesis, management and prognosis. Cardiovasc Surg. 2000;8:1-9.

4. Kavarana MN, Pessin-Minsley MS, Urtecho J, Catanese KA, Flannery M, $\mathrm{Oz} \mathrm{MC}$, et al. Right ventricular dysfunction and organ failure in left ventricular assist device recipients: a continuing problem. Ann Thorac Surg. 2002;73: 745-50.

5. Haneya A, Philipp A, Diez C, Metterlein T, Puehler T, Hilker M, et al. Successful use of temporary right ventricular support to avoid implantation of biventricular long-term assist device: a transcutaneous approach. ASAIO J. 2011;57:274-7.

6. Fitzpatrick JR III, Frederick JR, Hiesinger W, Hsu VM, McCormick RC, Kozin ED, et al. Early planned institution of biventricular mechanical circulatory support results in improved outcomes compared with delayed conversion of a left ventricular assist device to a biventricular assist device. J Thorac Cardiovasc Surg. 2009;137:971-7.

7. Takeda K, Naka Y, Yang JA, Uriel N, Colombo PC, Jorde UP, et al. Outcome of unplanned right ventricular assist device support for severe right heart failure af ter implantable left ventricular assist device insertion. J Heart Lung Transplant. 2014;33:141-8.

8. Samuels LE, Shemanski KA, Casanova-Ghosh E, Hagan K, Garwood P, Droogan C. Hybrid Ventricular Assist Device: Heartmate XVE LVAD and Abiomed AB5000 RVAD. ASAIO J. 2008;54:332-4.

9. Shehab S, Newton PJ, Allida SM, Jansz PC, Hayward CS. Biventricular mechanical support devices-clinical perspectives. Expert Rev Med Devices. 2016;13: $353-65$.

10. Takayama H, Truby L, Koekort M, Uriel N, Colombo P, Mancini DM, et al. Clinical outcome of mechanical circulatory support for refractory cardiogenic shock in the current era. J Heart Lung Transplant. 2013;32:106-11.

11. Sauer CM, Yuh DD, Bonde P. Extracorporeal membrane oxygenation use has increased by $433 \%$ in adults in the United States from 2006 to 2011. ASAIO J. 2015;61:31-6.

12. Ochiai Y, McCarthy PM, Smedira NG, Banbury MK, Navia JL, Feng J, et al. Predictors of severe right ventricular failure after implantable left ventricular assist device insertion: analysis of 245 patients. Circulation. 2002;106(12 suppl 1): I-198-202.

13. Baumwol J, Macdonald PS, Keogh AM, Kotlyar E, Spratt P, Jansz P, et al. Righ heart failure and "failure to thrive" after left ventricular assist device: clinical predictors and outcomes. J Heart Lung Transplant. 2011;30:888-95.

14. De Silva RJ, Soto C, Spratt P. Extra corporeal membrane oxygenation as right heart support following left ventricular assist device placement: a new cannulation technique. Heart Lung Circ. 2012;21:218-20. 
15. Shehab S, Macdonald PS, Keogh AM, Kotlyar E, Jabbour A, Robson D, et al. Chronic biventricular HVAD support-case series of right atrial and right ventricular implantation outcomes. J Heart Lung Transplant. 2016;35:466-73.

16. Lalonde SD, Alba AC, Rigobon A, Ross HJ, Delgado DH, Billia F, et al. Clinical differences between continuous flow ventricular assist devices: a comparison between HeartMate II and HeartWare HVAD. J Card Surg. 2013;28:604-10.

17. Wieselthaler GM, Gerry O, Jansz P, Khaghani A, Strueber M, Investigators HC. Initial clinical experience with a novel left ventricular assist device with a magnetically levitated rotor in a multi-institutional trial. J Heart Lung Transplant. 2010;29:1218-25.

18. Kirklin JK, Naftel DC, Kormos RL, Stevenson LW, Pagani FD, Miller MA, et al. Fifth INTERMACS annual report: risk factor analysis from more than 6,000 mechanical circulatory support patients. J Heart Lung Transplant. 2013;32:141-56.

19. Kirsch M, Vermes E, Damy T, Nakashima K, Sénéchal M, Boval B, et al. Singlecentre experience with the Thoratec paracorporeal ventricular assist device for patients with primary cardiac failure. Arch Cardiovasc Dis. 2009;102:509-18.

20. Brehm K, Heilmann C, Siepe M, Benk C, Beyersdorf F, Schlensak C. Thoratec paracorporeal biventricular assist device therapy: the Freiburg experience. Eur J Cardiothorac Surg. 2012;41:207-12.

21. Schweiger M, Schrempf J, Sereinigg M, Prenner G, Tscheliessnigg KH, Wasler A, et al. Complication profile of the Berlin heart EXCOR biventricular support in children. Artif Organs. 2013;37:730-5.

22. Saeed D, Maxhera B, Kamiya H, Lichtenberg A, Albert A. Alternative right ventricular assist device implantation technique for patients with perioperative right ventricular failure. J Thorac Cardiovasc Surg. 2015;149:927-32.
23. Boulate D, Luyt C-E, Pozzi M, Niculescu M, Combes A, Leprince P, et al. Acute lung injury after mechanical circulatory support implantation in patients on extracorporeal life support: an unrecognized problem. Eur J Cardiothorac Surg. 2013;44:544-50.

24. Alba AC, Rao V, Ivanov J, Ross HJ, Delgado DH. Usefulness of the INTERMACS scale to predict outcomes after mechanical assist device implantation. $J$ Heart Lung Transplant. 2009;28:827-33.

25. Rockett SR, Bryant JC, Morrow WR, Frazier EA, Fiser WP, McKamie WA, et al. Preliminary single center North American experience with the Berlin Heart pediatric EXCOR device. ASAIO J. 2008;54:479-82.

26. Gray BW, Haft JW, Hirsch JC, Annich GM, Hirschl RB, Bartlett RH. Extracorporeal life support: experience with 2000 patients. ASAIO J. 2015;61:2.

27. Paden ML, Conrad SA, Rycus PT, Thiagarajan RR. Extracorporeal life support organization registry report 2012. ASAIO J. 2013;59:202-10.

28. Drakos SG, Janicki L, Horne BD, Kfoury AG, Reid BB, Clayson S, et al. Risk factors predictive of right ventricular failure after left ventricular assist device implantation. Am J Cardiol. 2010;105:1030-5.

29. Shafii AE, Chamogeorgakis T, Mountis M, Gonzalez-Stawinski G. Fate of retained right ventricular assist device outflow grafts after right ventricular recovery. J Heart Lung Transplant. 2012;31:672-3.

Key Words: biventricular assist device, left ventricular assist device, venopulmonary arterial extracorporeal life support, advanced heart failure, temporary right ventricular support

Readers who found these articles interesting may also like to read the following papers found in recent and future issues of our sister publications, Seminars in Thoracic and Cardiovascular Surgery and Operative Techniques in Thoracic and Cardiovascular Surgery!

\section{Adult: Mechanical Circulatory Support}

STATE OF THE ART: Less Invasive Surgical Approaches for Left Ventricular Assist Device Implantation. Marcel Ricklefs. Semin Thoracic Surg 2018: 1-6

ORIGINAL SUBMISSION: Implantation of the HeartMate 3 -Description of the Surgical Technique. Friedhelm Beyersdorf. Oper Tech Thorac Cardiovasc 2018: In press

ORIGINAL SUBMISSION: Greater Omentum Wrapping to Treat Systemic Vad Infections. Sebastian Schulte Eistrup. Oper Tech Thorac Cardiovasc 2018: In press 